\title{
Eye movements in auditory space perception
}

\author{
BILL JONES \\ University of Waterloo, Waterloo, Ontario, Canada \\ and \\ BORIS KABANOFF \\ University of Queensland, St. Lucia, Brisbane, Queensland, Australia
}

\begin{abstract}
The hypothesis tested was that reports of auditory position are in part determined by target-directed eye movement. Using a signal recognition paradigm, we show that sensitivity to the position right or left of a tone decreases when the subject keeps his eyes fixed (Experiment I). We also show (Experiments II and III) that sensitivity declines considerably if the subject's eye movement is cues away from the tone either by a light source or by an instruction to the subject. Since providing the subject with a tactile spatial cue does not bias reports of auditory position (Experiment III), we argue that eye movement serves to update and stabilize auditory position memory. Finally, we argue that voluntary movement rather than a visual map (Warren, 1970) is likely to provide the framework for spatial judgments.
\end{abstract}

Traditionally, it has been argued that vision provides necessary referents for structuring auditory space (e.g., von Senden, 1960). Recently, Warren (1970) has shown that adults can point to the source of a sound more accurately in the light than in the dark, and he has argued that auditory inputs are organized in terms of a primary visual "map." Jones (1972), in contrast, argues that intersensory effects depend upon anatomical linkages of the different sensory areas via the motor cortex, which may serve to integrate neural activity by sampling the state of the different sensory receptors (Pribram, 1971). Space perception could depend, therefore, on a fusion of sensory modalities with vision as one channel in a reciprocal system rather than as a necessary reference point. The essential spatial framework would be given by motor activity and all sensory stimulation would map onto a pattern of movements. Conceivably, eye movements are a general means of updating and stabilizing position memory (Kinchla \& Allan, 1969) and, more specifically, eye movements may be involved in auditory localization since Fishman and Michael (1973) have shown that there are cells in the cat's visual cortex, responsive to both visual and auditory stimuli, which are probably linked to oculomotor nuclei.

If eye movements toward an auditory target can update auditory position memory, any considerable "noise" in the eye-movement control system would interfere with auditory localization. There is, in fact, considerable evidence that eye movements are "noisy" in the dark. Eye control in the dark is subject to involuntary drift (Becker \& Klein, 1973; Matin, Pearce, Matin, \& Kibler, 1966), which can result in

Address for reprints: Bill Jones. Department of Psychology, Carleton University, Ottawa, Ontario, Canada. errors of between $2^{\circ}$ and $5^{\circ}$ in repeating even a well-practiced movement (Becker \& Klein, 1973; Merton, 1961). The eyes may drift as much as $5^{\circ}$ to $10^{\circ}$ before the subject is consciously aware of the need for correction.

Such differences between eye control in the light and dark could explain Warren's (1970) finding that auditory localization is more accurate in the light. Possibly, eye drift away from the auditory target (Warren used a number of fairly closely spaced targets) could add a variable source of error in the dark which is not present in the light, i.e., subjects could rely upon knowledge of final eye position as a cue for auditory position. More likely, however, since subjects in the dark do not have precise knowledge of eye movement, since drift is both involuntary and largely unconscious, they will rely upon targetdirected eye movements to stabilize position memory. Such movements would be less accurate in the dark because they are superimposed upon a pattern of "noisy" drift. Platt and Warren (1972) have, in fact, shown that auditory localization in the light is no more accurate than in the dark if the subject does not make eye movements. The importance of visual afference for auditory localization may, therefore, be that of stabilizing eye drift rather than that of providing primary reference points for auditory cues.

The present experiments concentrate on the all-or-none effects of eye-movement involvement in auditory localization. We assume that auditory position is given initially from aural cues and that auditory position memory is stabilized by a target-directed eye movement. If auditory position is given in part from voluntary eye movements, localization should be less accurate when the person makes no eye movements and considerably less accurate if eye movements are directed away from the 
auditory target.

In all the following experiments, subjects had to report the position right or left of a sound or of a light source. Right signals were arbitrarily treated as "signal" and left signals as "noise," and a curve of receiver-operating characteristics (ROC) was obtained using a rating scale procedure (Green \& Swets, 1966). The index of localization accuracy was the proportion of area under the ROC curve. This measure, designated $P(A)$, is a nonparametric index of sensitivity independent of bias (Green \& Swets, 1966).

\section{EXPERIMENT I}

Platt and Warren (1972) measured variable and absolute error when subjects pointed to one of a number of possible sound sources. The following psychophysical experiment aims to confirm their finding that auditory localization is less accurate without target-directed eye movements.

\section{Method}

Subjects. The subjects were six undergraduates, all of whom had normal uncorrected hearing and vision.

Apparatus. Experimentation was carried out in an enclosed, lighted room. Minimum ambient noise was approximately $30 \mathrm{~dB}$ above threshold. Each subject was seated in a comfortable, adjustable chair inside a metal framework $(2.00 \times 1.20 \times 0.35 \mathrm{~m})$. The position of the subject's head was stabilized by an adjustable band which encircled his head and which was fixed to the apparatus. Above the subject's head and hidden from his sight, two beams extended a distance of $1.25 \mathrm{~m}$. From each of the beams a small hearing-aid loudspeaker was suspended. The speakers were hidden from the subject's view by a thin opaque cloth screen. The position of each speaker was fixed at $3^{\circ}$ to the right and left of the midline of the subject's head. That was the minimum separation required to monitor the subject's eye movements on an oscilloscope screen. $\mathrm{Ag} / \mathrm{AgCl}$ electrodes were placed at the external canthus of each eye and movements of the eys in one direction or another were shown as deflections of a trace on a B.W.D. (Model 521) dual-beam oscilloscope with differential dc amplifier. A second trace indicated signal onset to allow accurate monitoring of the direction of eye movement.

Auditory signals were step-input tones, $0.01 \mathrm{sec}$ in duration, with a frequency of $3,200 \mathrm{~Hz}$ and an intensity level of $50 \mathrm{~dB}$ above threshold.

Procedure. There were two experimental conditions. In both, the subject was required to report the position, right or left, of the tone. On each trial, the tone occurred at random on one side or the other. In the "eyes fixed" condition, the subject was instructed to maintain a straight-ahead constant fixation of the eyes. In the "eyes free" condition. he was instructed to move his eyes in the perceived direction of the tone. The two conditions were a within-subject variable, counterbalanced ABBA in blocks of 150 trials. Prior to the experimental session, electrodes were fitted and the subject's eye movements were monitored on the oscilloscope so as to ensure that

Table 1

$P(A)$ Values Obtained in Experiment I

\begin{tabular}{ccc}
\hline Subject & Free & Fixed \\
\hline 1 & .87 & .67 \\
2 & .93 & .69 \\
3 & .94 & .74 \\
4 & .94 & .66 \\
5 & .88 & .65 \\
6 & .90 & .72 \\
\hline
\end{tabular}

he followed instructions.

The subjects indicated their reports of signal position on a scale from 1 ("complete confidence") through 2 ("fairly confident"), 3 ("uncertain"), to 4 ("very uncertain"). Since right-hand signals were considered as "signal" and left-hand signals as "noise," this procedure corresponded to an 8-point rating scale. In all conditions, the intertrial interval was $6 \mathrm{sec}$ and subjects were instructed to respond as quickly as possible.

\section{Results}

For each subject, an ROC curve was drawn for each condition (where necessary, rating-scale categories were collapsed) and $\mathrm{P}(\mathrm{A})$ was calculated (Table 1). Statistical comparison between conditions is hardly necessary. All subjects were more sensitive to the position of the tone when they were able to move their eyes. Differences were in fact significant, $t(5)=5.50$, $\mathrm{p}<.001$. It may be, therefore, that target-directed movements are a direct source of auditory position information.

\section{EXPERIMENT II}

If eye movements can be used to stabilize memory for auditory location, it should follow that cueing eye movements away from the auditory target would lead to inaccurate reports of position. In the following experiment, performance of subjects in the dark was compared without eye movements, with eye movements in the direction of the target, or with eye movements in the opposite direction. The movements were cued by means of a light source.

\section{Method}

Subjects. The subjects were five undergraduates, none of whom had taken part in Experiment $I$. All of the subjects had normal uncorrected vision and hearing.

Apparatus. The apparatus was that used in Experiment I, except that the cloth screen was removed from in front of the sound sources and a light source was placed immediately above each speaker. The light signals, $0.01 \mathrm{sec}$ in duration and $14 \mathrm{fL}$ in intensity, were produced by $12-\mathrm{V} 2-\mathrm{W}$ bulbs encased in a small black box with a $1-\mathrm{mm}$ aperture covered with a single strip of masking tape.

Procedure. The experiment took place in a totally darkened room. Electrodes were fitted outside the room and the subject was blindfolded. The blind fold was removed only when the room lights were turned out. The procedure ensured that the subject had no opportunity to view the apparatus and acquire a visual context.

There were two eye-movement conditions. In one, the subject was instructed to move his eyes in the direction of the light source (free); in the other, the subject was instructed to look straight ahead without moving his eyes (fixed). Within each eye-movement condition, signal presentation was varied such that on each trial the sound and light sources were either on the same side (congruent) or on opposite sides (discrepant). Onset of sound and light was simultaneous. Eye-movement and signal presentation were within-subject variables. Eye movements were counterbalanced in the same fashion for all subjects (fixed, free, free, fixed), and within each block of eye movements, signal presentation was randomized with the constraint that congruent and discrepant presentation appear equally often. Following signal presentation, the subject was required to report either the position of the tone or the position of the light using the rating-scale procedure. Since the experimenter asked for light or tone at random (with the constraint that the probability of light or tone was 0.5 ), the subject could not anticipate on any trial whether he was to report tone or light. For each signal 
presentation, the position of the signals was randomly varied, with an equal number of signal and noise trials.

Eye movements were monitored as in Experiment I, and trials on which the subject did not look in the direction of the light were replaced. In all, there were 1,200 trials, i.e., for each level of the design there were 75 signal and 75 noise trials.

\section{Results and Discussion}

As for Experiment I, P(A) values were obtained for each subject under each condition (Table 2). To minimize skew and facilitate analysis of variance, $P(A)$ values were conventionally transformed as 2 arc sine $\sqrt{ } \mathrm{P}(\mathrm{A})$. A $2 \times 2 \times 2$ ANOVA of the resulting values, with eye movement, spatial presentation, and modality reported conditions as within-subject variables, showed that the main effect of eye movements did not reach significance, $F(1,4)=4.69$, while the effects of signal presentation conditions, $\mathrm{F}(1,4)=60.12, \mathrm{p}<.003$, and modality reported, $\mathrm{F}(1,4)=57.62, \mathrm{p}<.003$, were both highly significant. The Signal Presentation by Modality Reported interaction was significant, $F(1,4)=8.06$, $\mathrm{p}<.05$, as was the three-way interaction, $F(1,4)=$ $80.22, p<.002$. Neither the Eye Movement by Signal Presentation interaction, $F(1,4)=1.03$, nor the Eye Movement by Modality Reported interaction, $F(1,4)$ $=1.69$, reached significance.

Duncan's multiple range test showed that, with eyes fixed, differences between localization of the light and the tone were significant $(p<.01)$ for both congruent and discrepant presentation. Again with eyes fixed, differences between congruent and discrepant presentation for both light and tone were significant $(p<.01)$. In other words, accuracy of localization of both light and tone is a function of spatial identity. It seems reasonable to suppose that presentation of both stimuli on the same side results in processing along a common decision axis, while spatially distinct signals are processed as independent decisions. Formally, the addition of two signals of equal signal-to-noise power, provided that signals are correlated and noise uncorrelated, would result in four times the signal power and only twice the noise power (Green \& Swets, 1966).

With eyes free, Duncan's test showed significant differences $(p<.01)$ between localization of the light and the tone in both the congruent and discrepant conditions. Additionally, differences in localization of the tone, with eyes free, between the congruent and discrepant conditions were significant $(p<.01)$.

Duncan's test also showed that differences between eyes fixed and eyes free localization of the tone were significant for both congruent and discrepant presentations $(\mathrm{p}<.01)$. Interestingly, differences between eyes fixed and eyes free localization of the light with discrepant presentation were also significant $(\mathrm{p}<.01)$, suggesting that eye movements are a useful source of visual position memory (see Kinchla \& Allan, 1969).
Table 2

P(A) Values Obtained in Experiment II

\begin{tabular}{|c|c|c|c|c|c|c|c|c|}
\hline \multirow{3}{*}{$\begin{array}{l}\text { Sub- } \\
\text { ject }\end{array}$} & \multicolumn{4}{|c|}{ Fixed } & \multicolumn{4}{|c|}{ Free } \\
\hline & \multicolumn{2}{|c|}{ Congruent } & \multicolumn{2}{|c|}{ Discrepant } & \multicolumn{2}{|c|}{ Congruent } & \multicolumn{2}{|c|}{ Discrepant } \\
\hline & $\mathbf{L}$ & $\mathbf{T}$ & $\mathbf{L}$ & $\mathbf{T}$ & L & $\mathbf{T}$ & $\mathbf{L}$ & $\mathrm{T}$ \\
\hline 1 & .99 & .78 & .68 & .37 & .99 & .85 & .96 & .21 \\
\hline 2 & .98 & .58 & .56 & .42 & .98 & .94 & .97 & .31 \\
\hline 3 & .91 & .56 & .56 & .47 & .93 & .70 & .95 & .39 \\
\hline 4 & .99 & .68 & .99 & .46 & .99 & .94 & .95 & .08 \\
\hline 5 & 1.00 & .52 & .99 & .35 & 1.00 & .92 & 1.00 & .22 \\
\hline
\end{tabular}

Contrary to Warren's (1970) notion that the visual environment structures auditory space, we have assumed that visual information may do no more than enable subjects to control their eye movements and hence more efficiently stabilize auditory position memory. The present results show that memory for visual position may also depend in part upon eye movements. Maximum accuracy of localization of the light requires either target-directed eye movements or the congruency of light and tone positions. In other words, the visual cue may not be the primary referent for auditory position since it is itself fixed in part from eye movement and in part from knowledge of the position of the tone. The relationship between auditory and visual cues seems, therefore, to be a reciprocal one, such that in other situations auditory cues could interact with eye movement to determine visual position.

\section{EXPERIMENT III}

Platt and Warren (1972) argued that a "textured visual environment" interacts with eye movements to determine auditory position. In Experiment II, subjects had to fix the position of visual and auditory signals without a visual background. Nevertheless, there was an interaction between visual, auditory, and movement cues. Since we have argued that accuracy of auditory localization depends upon eye-movement control rather than a visual "map," it is of interest to know whether eye movements could facilitate auditory localization in the absence of any visual cues. In the following experiment, we attempted to structure eye movements in the dark by simply asking the subject to move his eyes in one direction. Thus, instructions from the experimenter could "preprogram" the subject's voluntary eye movements.

\section{Method}

Subjects. The subjects were five undergraduates with normal hearing who had not taken part in either of the previous experiments.

Apparatus and Procedure. Except that the light sources were not used, the apparatus was that used in the previous experiments. The general procedure followed Experiments I and II. In the present experiment, a single tone was presented randomly to right or left on each trial. Congruent with signal presentation, the subject was instructed again at random to move his eyes to the right or to the 
leti upon hearing the tone. or to keep his eves fixed in front of him. Instructions were delivered automatically over a tape recorder. Consequently, eye movement was spatially congruent. spatially discrepant. or spatially neutral with respect to the signal. Trials on which the subject failed to carry out instructions were replaced. As in the previous experiment. the subject reported the position of the tone using the rating-scale procedure. For all three conditions (congruent. discrepant. fixed). there were 100 "signal" and 100 "noise" trials making 600 trials in all.

\section{Results and Discussion}

$P(A)$ values were obtained and transformed as above (Table 3). One-way analysis of variance with repeated measures of transformed values indicated a significant difference between conditions, $F(2.8)=$ $13^{-} .42 . p<.00002$. Duncan's multiple range test showed that all means differed from each other at the .01 level. The results again support the hypothesis that the direction of eye movements largely determines reports of auditory position even in the absence of information from the visual environment.

\section{EXPERIMENT IV}

One argument against our position is that subjects in Experiments II and III could form some general cognitive bias to respond that the auditory signal was in the same direction as the light or the instruction to move the eves. We have argued, following Kinchla and Allan (1969), that eye movements systematically bias auditory position memory. They point out (p. 544) that such a bias would have the same effect on performance as a systematic but opposite shift in response bias during the period from onset of the signal to completion of the eye movement. Formally. the two effects would be impossible to distinguish since both would yield the same final performance measure. In the final experiment, we attempted to bias reports of auditory position using cognitive information independent of eye movements. Specifically. we provided subjects with a tactual cue that was either congruent or discrepant with the position of the tone. If subjects without eye movements formed the hypothesis that auditory position was at least partially correlated with the tactual cue. it may be that the procedures used in Experiments II and III did no more than introduce a general cognitive bias. On the other hand, if performance was not affected by the tactual cue, the memory stabilization theory of eye movements would appear stronger. Tactual signals were electric shocks delivered to the back of the subject's right or left shoulder. These points were chosen in an attempt to make judgments of tactual position somewhat difficult (see. e.g., Halnan \& Wright. 1960).

\section{Method}

Subjects. The subjects were eight undergraduates who had not taken part in any of the previous experiments.

Apparatus. The same apparatus was used as in Experiment I. except that electrical contacts were fixed to a point below each of the subject's shoulder blades. An electric shock. $.01 \mathrm{sec}$ in
Table 3

P(A) Values Obtained in Experiment III

\begin{tabular}{lccccc}
\hline & \multicolumn{5}{c}{ Subject } \\
\cline { 2 - 6 } Condition & 1 & 2 & 3 & 4 & 5 \\
\hline Congruent & .74 & .75 & .77 & .70 & .73 \\
Discrepant & .38 & .37 & .38 & .36 & .43 \\
Fixed & .57 & .62 & .53 & .50 & .64 \\
\hline
\end{tabular}

duration. from a $5 . V$ battery was delivered simultaneously with the auditory signal.

Procedure. The general procedure for monitoring eye movement followed previous experiments. The subject in the present experiment tried to maintain a constant straight-ahead fixation throughout. In two conditions. the electric shock was presented either on the same side (congruent) or on the side opposite to the tone (discrepant). In the remaining condition, the tone was presented alone. On each trial. one of the three conditions was presented in a random sequence. The position of the tone on each trial was also randomized, with the constraint that right and left occur equally often in each condition. On half the trials, again at random, the subject was asked to report the position of the tone. and on the remainder. the position of the shock. In all, there were 600 trials.

\section{Results}

$P(A)$ values were obtained as before (Table 4). Recognition of the position of the shock was almost perfect for all subjects and is not reported in the table. Inspection of the table indicates that subjects do not differ in accuracy of localization in any of the three conditions. One-way between-subjects analysis of variance showed no significant differences between conditions, $F(2.4)=1.28$. Merely providing the subject with a spatial signal does not bias his reports of auditory position. It seems reasonable to argue. therefore. that target-direct eye movements do more than merely cue a response bias and may, as we have argued. stabilize position memory.

\section{GENERAL DISCUSSION}

Using a different paradigm, our results (Experiment I) directly confirm Platt and Warren's (1972) hypothesis that accuracy of auditory localization is increased if the subject moves his eyes in the direction of the target. Since other spatial cues do not necessarily bias reports of auditory position (Experiment IV), voluntary eye movements may be part of the operations by which the person updates position memory.

Table 4

P(A) Value for Recognition of the Tone in Experiment IV

\begin{tabular}{cccc}
\hline Subject & Congruent & Discrepant & Tone \\
\hline 1 & .58 & .58 & .57 \\
2 & .64 & .58 & .62 \\
3 & .58 & .59 & .63 \\
4 & .66 & .68 & .68 \\
5 & .68 & .53 & .59 \\
6 & .50 & .49 & .52 \\
7 & .66 & .68 & .64 \\
8 & .54 & .52 & .60 \\
\hline
\end{tabular}


On the other hand, there seems no need to argue that visual cues are the primary reference or map for auditory position (Warren, 1970). Experiment II shows something of the interaction of visual, auditory, and movement cues in spatial perception. Conceivably, people may use any and whatever cues are available to determine judgments of position. We would argue that the framework for such judgments is likely to be voluntary eye movements since eye movements are known but not perceived in the sense that eye-movement control probably depends upon central monitoring of efferent signals rather than feedback from proprioceptors in the eye muscles (see, e.g., Festinger \& Canon, 1965; Matin, Matin, \& Pola, 1970; Skavenski, Haddad, \& Steinman, 1972). The person may be able to use voluntary eye movements to structure spatial judgments precisely because they are something he does and not something he has to separately perceive. Our research has been mainly concerned to show the direct effects of voluntary eye movements in auditory judgments. Other research (e.g., Kinchla \&Allan, 1969; Skavenski et al., 1972) has shown the importance of such movements in determining visual position as does, in part, Experiment II. Therefore, eye movement may provide a general reference for all spatial judgments.

\section{REFERENCES}

BECKER, W., \& KLEIN, H. M. Accuracy of saccadic eye movements and maintenance of eccentric eye positions in the dark. Vision Research, 1973, 13, 1021-1034.
Festinger, L., \& Canon, L. K. Information about spatial location based on knowledge about efference. Psychological Review, 1965, 72, 373-384.

Fishman, M. C., \& Michael, C. R. Integration of auditory information in the cat's visual cortex. Vision Research, 1973, 13, $1415-1419$.

Green, D. M., \& Swets, J. A. Signal detection theory and psychophysics. New York: Wiley, 1966.

Halnan, C. R. E., \& WRight, G. H. Tactile localization. Brain, 1960, 83, 677-700.

JoNEs, B. Facilitation of visual perception through voluntary movement in elementary schoolchildren. Journal of Experimental Child Psychology, 1972, 14, 408-415.

Kinchla, R. A., \& Allan, L. G. A theory of visual movement perception. Psychological Review, 1969, 76, 537-558.

Matin, L. M., Matin, E., \& Pola, J, Visual perception of direction when voluntary saccades occur. Perception \& Psychophysics, 1970, 8, 9-14.

Matin, L., Pearce, D., Matin, E., \& Kibler, G. Visual perception of direction in the dark: Roles of local sign, eye movements and ocular proprioception. Vision Research, $1966,6,453-469$.

Merton, P. A. The accuracy of directing the eyes and the hand in the dark. Journal of Physiology, London, 1961, 156, 555-577.

Platt, B. B., \& Warren, D. H. Auditory localization: The importance of eye movements and a textured visual environment. Perception \& Psychophysics, 1972, 12, 245-248.

Pribram, K. H. Languages of the brain. New York: PrenticeHall, 1971.

voN SENDEN, M. Space and sight. London: Methuen, 1960.

Skavenski, A. A., Haddad, G., \& Steinman, R. The extraretinal signal for the visual perception of direction. Perception \& Psychophysics, 1972, 11, 287-290.

WARREN, D. H. Intermodality interactions in spatial localization. Cognitive Psychology, 1970, 1, 114-133.

(Received for publication April 18, 1974; revision received October $25,1974$. 